\title{
Multiple Human Papilloma Virus Infections and Their Impact on the Development of High-Risk Cervical Lesions
}

\author{
Katrina L. Salazar ${ }^{a}$ Haijun Steve Zhou ${ }^{a}$ Jiaqiong Xu ${ }^{b}$ Leif E. Peterson ${ }^{b}$ \\ Mary R. Schwartz ${ }^{a}$ Dina R. Mody ${ }^{a, c}$ Yimin Ge ${ }^{a, c}$ \\ ${ }^{a}$ Department of Pathology and Genomic Medicine, and ${ }^{\mathrm{b}}$ Center for Biostatistics, Houston Methodist Hospital and \\ Research Institute, Houston, Tex., and 'Weill Medical College of Cornell University, New York, N.Y., USA
}

\section{Key Words}

Cervical cancer - Cervical dysplasia - Human papillomavirus . Intergenotypic interaction - Multiple human papillomavirus infections

\begin{abstract}
Objective: Individuals are often infected with multiple genotypes of human papillomavirus (HPV) simultaneously, but the role these infections play in the development of cervical disease is not well established. This study aimed to determine the association of multiple HPV infections with highrisk cervical lesions (hrCLs). Study Design: HPV genotyping was performed on 798 SurePath specimens collected between December 1, 2009, and April 30, 2011. The cases were classified as $\mathrm{hrCL}(\mathrm{n}=90)$ or non-hrCL $(\mathrm{n}=708)$ based on cytology diagnoses. The association between $\mathrm{hrCL}$ and HPV infection patterns was analyzed. Results: Multiple HPV infections were frequently encountered (38.2\%) in the cohort. Increased frequency of hrCLs was associated with a single high-risk HPV (hrHPV) infection. An additive or synergistic effect was not observed for hrCL in multiple HPV infections. The $\mathrm{hrCL}$ rates appeared to decrease in various patterns of multiple HPV infections, but the reduction was not statistically significant. Conclusions: Multiple HPV infections are
\end{abstract}

common with no additive or synergistic effect on the development of hrCL. Conversely, reduced hrCL rates were observed in various patterns of multiple HPV infections compared to their single-genotype infection counterparts, suggestive of possible intergenotypic competition or more effective immune response triggered by multiple infections. Further studies in larger cohorts are needed.

(c) 2015 S. Karger AG, Base

\section{Introduction}

Human papillomaviruses (HPVs) are small, nonenveloped, double-stranded DNA viruses, and more than 40 genotypes have been associated with infection of the female genital tract. Numerous studies have focused on infections due to various high-risk HPV (hrHPV) genotypes and their effect on the development of cervical dysplasia and cancers [1]. Based on the oncogenic potential of individual HPV genotypes, the expert working group of the International Agency for Research on Cancer (IARC) classified HPV into four groups: carcinogenic (group 1), probably carcinogenic (group 2A), possibly carcinogenic (group 2B) and not classifiable (group 3) [2].

\section{KARGER 125}

(c) 2015 S. Karger AG, Base

0001-5547/15/0595-0391\$39.50/0

E-Mail karger@karger.com

www.karger.com/acy
Correspondence to: Dr. Yimin Ge

Department of Pathology and Genomic Medicine

Houston Methodist Hospital

6565 Fannin Street, Suite M227, Houston, TX 77030 (USA)

E-Mail yge@ houstonmethodist.org 
Results from various study populations have shown that multiple HPV genotype infections are frequently encountered, and cervical infections are not always caused by a single HPV genotype [3-12]. Despite mounting reports on the outcomes of infection with an individual hrHPV genotype, the interactions between co-infecting HPV genotypes are not well studied, and the effect of multiple HPV infections on cervical disease is controversial. Several studies have demonstrated that multiple HPV infections are a significant risk factor for high-risk cervical lesions (hrCLs) [13-19]. These reports also concluded that HPV infections are linearly correlated with the severity of cervical intraepithelial neoplasia, and that the infections appear to act synergistically in cervical carcinogenesis. Conversely, alternate studies showed that multiple HPV infections have no synergistic or additive effect on the development of cervical diseases or increased risk for hrCL as compared to a single HPV infection [9, 20-24]. The findings from a more recent study, however, suggest that competitive and/or cooperative interactions may exist among certain HPV genotypes [10]. In this context, Sobota et al. [25] showed that co-infection with HPV genotypes from the same taxonomic species reduced the likelihood of progression to hrCL in HIV-positive women; co-infection with HPV genotypes from different species, however, did not confer protection from hrCL.

In view of the contradicting data reported to date, additional studies are warranted to gain mechanistic insight and elucidate the effect of multiple HPV infections on cervical dysplasia and cancer. In this study, we analyzed the HPV infection patterns in an underserved population and their association with hrCLs. The aim of the study was to determine the effect of multiple HPV infections on cervical dysplasia and cancer, and to explore the possible interactions among the HPV genotypes in women with multiple HPV infections.

\section{Materials and Methods}

Study Population and Cytology Classification

The study was conducted with approval from the Institutional Review Board of the Houston Methodist Research Institute (IRB1210-0221, approved February 25, 2011). The study included 798 women who were referred to the Houston Methodist Hospital from charity clinics in the Houston area for abnormal Papanicolaou tests from December 1, 2009, to April 30, 2011. The study population was comprised of predominantly Hispanic women who had no medical insurance and did not qualify for Medicare or Medicaid programs at the time of the study.

Liquid-based Papanicolaou tests (SurePath; BD, Franklin Lakes, N.J., USA) were performed for cytological evaluation, and the findings were interpreted according to the criteria set by the 2001 Bethesda System for cervicovaginal cytology reporting. All abnormal Papanicolaou tests were interpreted by board-certified cytopathologists and gynecologic pathologists. Based on cytological diagnoses, the cases were divided into two general groups: those with hrCLs (90 cases), including atypical squamous cells, cannot exclude high-grade squamous intraepithelial lesion (ASC-H), lowgrade squamous intraepithelial lesion with atypical cells, cannot exclude high-grade squamous intraepithelial lesion (LSIL-H) and high-grade squamous intraepithelial lesion (HSIL), and those with non-hrCL (708 cases), including atypical squamous cells of undetermined significance (ASC-US), low-grade squamous intraepithelial lesion (LSIL), and negative for intraepithelial lesion or malignancy (NILM).

HPV Genotyping by DNA Microarray and DNA Sequencing

HPV DNA was extracted from the residual SurePath specimens and amplified by polymerase chain reaction in the $\mathrm{L} 1$ region of the HPV genome before being labeled with Cy5 and hybridized with an HPV DNA microarray chip with $40 \mathrm{HPV}$ genotypes (GG HPV DNA genotyping chip kit; GoodGene Inc., Seoul, Korea). The signal was visualized using a GenePix 4000B microarray scanner (Molecular Devices Inc., Sunnyvale, Calif., USA). The detection limit of the HPV 40 DNA microarray chip is between 10 and 100 copies of HPV DNA per sample. The microarray data were confirmed by direct DNA sequencing. The data obtained by automated DNA sequencing were analyzed for HPV genotypes using BLAST (Basic Local Alignment Search Tool) search (http://www. ncbi.nlm.nih.gov/BLAST/). DNA sequencing identified the most dominant genotype in a given specimen and served as a confirmatory assay.

\section{Data Analysis}

The HPV genotypes in IARC groups 1 and $2 \mathrm{~A}$ are commonly referred to as hrHPV, and include genotypes 16, 18, 31, 33, 35, 39, $45,51,52,56,58,59$ and $68 \mathrm{~A} / 68 \mathrm{~B}$. In this study, IARC groups $2 \mathrm{~B}$ and 3 are collectively referred to as non-hrHPV. Odds ratios (ORs) and 95\% confidence intervals (CIs) were calculated, and Fisher's exact test was used to determine the significance of the differences in $\mathrm{hrCL}$ rates among the various HPV infection patterns. All analyses were performed with STATA version 13 (StataCorp LP, College Station, Tex., USA). Statistical significance was defined as two-tailed $\mathrm{p}<0.05$ for all tests.

\section{Results}

The average age of the patients was 36.5 years (range 19-85 years). The majority of the patients were between 20 and 39 years old $(n=539 ; 68 \%)$, which was also the dominant age group for abnormal cytology diagnoses. In the cytological diagnosis groups, hrHPV genotypes were found in $67 \%$ of women with hrCL and $38 \%$ of those with non-hrCL.

The prevalence of hrHPV and non-hrHPV among the cytological diagnosis groups is shown in figure 1. A higher proportion of hrCL was seen in women infected 


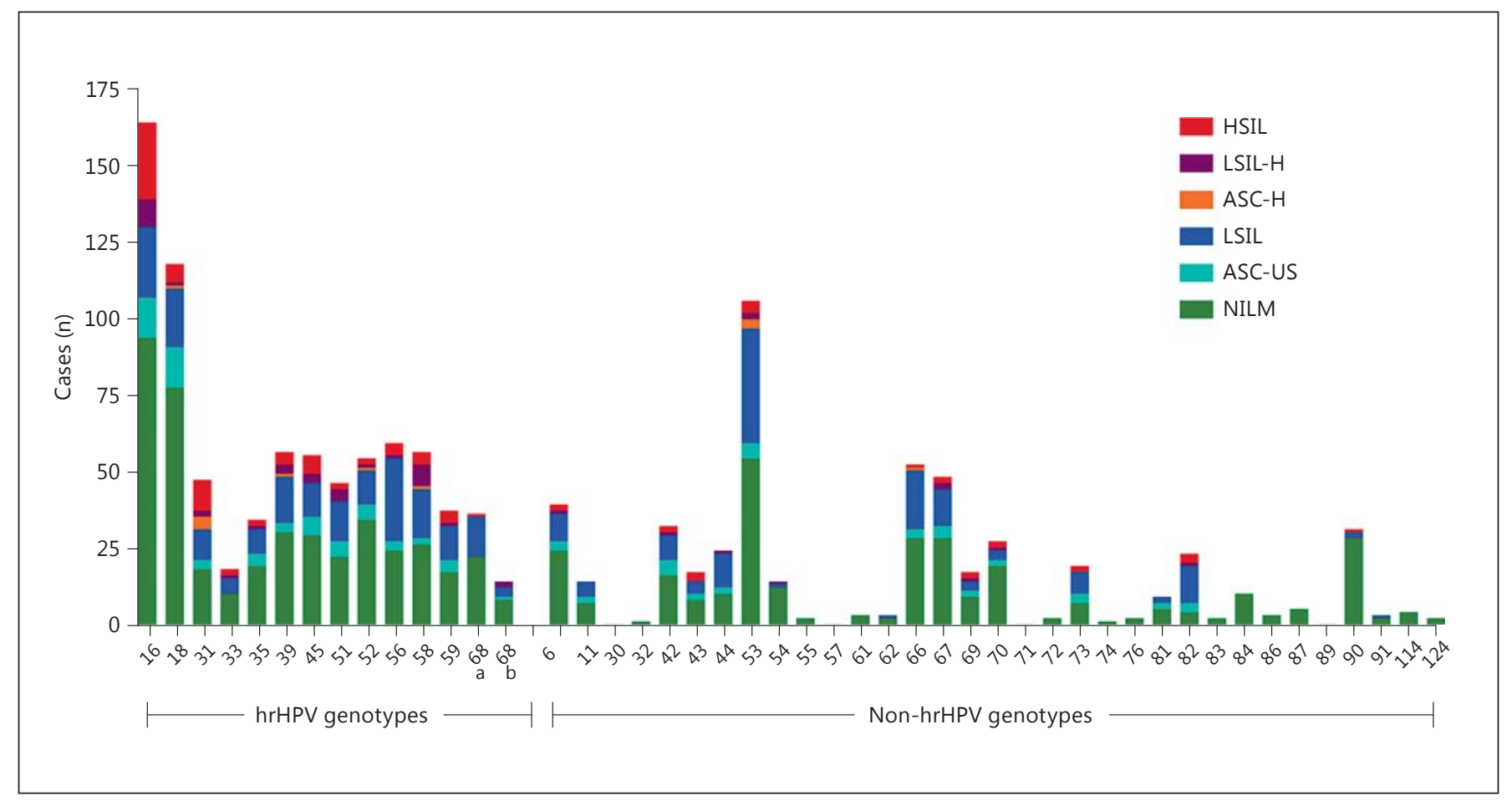

Fig. 1. HPV genotypic prevalence in cytology diagnosis categories.

with hrHPV genotypes such as HPV 16, 31 and 45. The HPV infection status (single, multiple or negative) among age groups is shown in figure 2. Multiple HPV infections were observed in $38.2 \%$ of the women, whereas $54.9 \%$ of the women had a single HPV infection; no HPV was detected in $7 \%$ of the cases. The frequency of multiple HPV infection reached a maximum in the age range from 20 to 29 years and gradually decreased thereafter.

The comparison of HPV infection patterns (single or multiple) between cytology groups of hrCL and nonhrCL is shown in table 1. When all HPV genotypes were considered, the rates of hrCL were the same in women with a single or multiple HPV infections.

Table 2 summarizes the cytology results and statistics among various HPV infection patterns. In women with a single HPV infection, the hrCL rate was strongly associated with a single genotype infection of hrHPV as compared to those infected with a single non-hrHPV genotype $(\mathrm{OR}=7.28,95 \% \mathrm{CI}: 2.99-21.27, \mathrm{p}<0.0001)$ or those in the HPV-negative group (OR $=6.16,95 \% \mathrm{CI}$ : $1.45-$ $26.19, \mathrm{p}=0.004)$. In women infected with multiple HPV genotypes, hrCL was significantly associated with multiple genotypes of hrHPV compared to those infected

Impact of Multiple HPV Infections

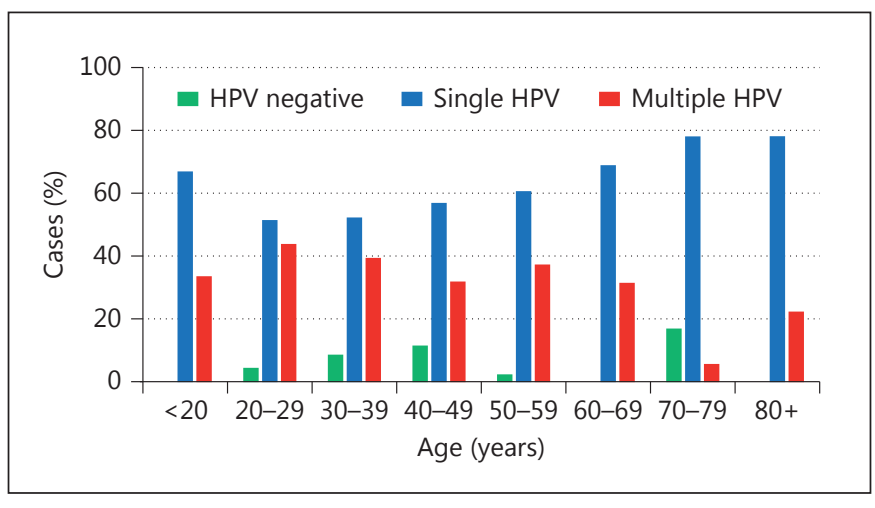

Fig. 2. HPV infection patterns among age groups.

Table 1. Single and multiple HPV infections in cytology groups

\begin{tabular}{llll}
\hline $\begin{array}{l}\text { Cytology } \\
\text { groups }\end{array}$ & $\begin{array}{l}\text { HPV } \\
\text { negative }\end{array}$ & $\begin{array}{l}\text { Single } \\
\text { HPV }\end{array}$ & \multicolumn{1}{l}{$\begin{array}{l}\text { Multiple } \\
\text { HPV }\end{array}$} \\
\hline $\begin{array}{l}\text { Non-hrCL } \\
\text { hrCL }\end{array}$ & $\begin{array}{c}53(96 \%) \\
2(4 \%)\end{array}$ & $\begin{array}{rlr}386(88 \%) \\
52(12 \%)\end{array}$ & $\begin{array}{r}269(88 \%) \\
36(12 \%)\end{array}$ \\
\hline
\end{tabular}

hrCL = ASC-H, LSIL-H and HSIL. 
Table 2. Patterns of multiple HPV infections in cytology groups

\begin{tabular}{|c|c|c|c|c|c|c|}
\hline \multirow[t]{2}{*}{ Cytology group } & \multirow{2}{*}{$\begin{array}{l}\text { HPV } \\
\text { negative }\end{array}$} & \multicolumn{2}{|l|}{ Single HPV } & \multicolumn{3}{|l|}{ Multiple HPV } \\
\hline & & hrHPV & non-hrHPV & hrHPV & non-hrHPV & $\mathrm{hr}+$ non-hrHPV \\
\hline Non-hrCL & $53(96 \%)$ & $198(81 \%)$ & $188(97 \%)$ & $73(84 \%)$ & $38(97 \%)$ & $158(88 \%)$ \\
\hline OR (95\% CI) & & $6.16(1.45-26.19)$ & $0.85(0.17-4.31)$ & $5.08(1.11-23.31)$ & $0.7(0.06-7.97)$ & $3.52(0.80-15.52)$ \\
\hline $\mathrm{p}$ value & & 0.004 & 1 & 0.03 & 1 & 0.12 \\
\hline
\end{tabular}

hrCL = ASC-H, LSIL-H and HSIL. ORs (95\% CIs) were calculated with HPV negative as the reference group.

Table 3. Number of co-infecting hrHPV genotypes in cytology groups

\begin{tabular}{lccccc}
\hline Cytology group & HPV negative & 1 hrHPV & 2 hrHPV & $3+$ hrHPV & Non-hrHPV \\
\hline hrCL & $2(4 \%)$ & $46(19 \%)$ & $11(18 \%)$ & $3(11 \%)$ & $7(3 \%)$ \\
Non-hrCL & $53(96 \%)$ & $198(81 \%)$ & $49(82 \%)$ & $24(89 \%)$ & $226(97 \%)$ \\
OR $(95 \%$ CI $)$ & & $6.16(1.45-26.19)$ & $5.95(1.26-28.19)$ & $3.31(0.52-21.13)$ & $0.82(0.17-4.06)$ \\
p value & & 0.004 & 0.02 & 0.33 & 0.68 \\
\hline
\end{tabular}

hrCL = ASC-H, LSIL-H and HSIL. ORs (95\% CIs) were calculated with HPV negative as the reference group.

Table 4. Infection patterns associated with HPV 16/18 and non-16/18 genotypes

\begin{tabular}{lccccc}
\hline Cytology group & HPV negative & HPV 16/18 & $\begin{array}{l}\text { Single non-16/18 } \\
\text { hrHPV }\end{array}$ & $\begin{array}{l}\text { HPV 16/18 }+ \\
\text { non-16/18 hrHPV }\end{array}$ & $\begin{array}{l}\text { HPV 16/18 }+ \\
\text { non-hrHPV }\end{array}$ \\
\hline hrCL & $2(4 \%)$ & $23(20 \%)$ & $24(17 \%)$ & $13(13 \%)$ & $2(6 \%)$ \\
Non-hrCL & $53(96 \%)$ & $92(80 \%)$ & $114(83 \%)$ & $89(87 \%)$ & $29(94 \%)$ \\
OR (95\% CI) & & $6.63(1.5-29.22)$ & $5.58(1.27-24.48)$ & $3.87(0.84-17.82)$ & $1.83(0.24-13.66)$ \\
p value & & 0.005 & 0.01 & 0.09 & 0.62 \\
\hline
\end{tabular}

hrCL = ASC-H, LSIL-H and HSIL; HPV 16/18 = HPV 16 and/or 18. ORs (95\% CIs) were calculated with HPV negative as the reference group.

with multiple non-hrHPV genotypes $(\mathrm{OR}=7.29$, CI: $1.02-316.06, \mathrm{p}=0.04)$ or those in the HPV-negative group $(\mathrm{OR}=5.08,95 \% \mathrm{CI}: 1.11-23.31, \mathrm{p}=0.03)$. There were no statistically significant differences in hrCL rates when comparing other HPV infection patterns, including 1 versus $2 \mathrm{hrHPV}$ genotypes, 1 versus 2 non-hrHPV genotypes, or non-hrHPV versus HPV negative. However, in women infected with combined hrHPV and nonhrHPV genotypes, the hrCL rate was somewhat reduced and more similar to those infected with non-hrHPV genotypes $(\mathrm{OR}=5.05,95 \% \mathrm{CI}: 0.66-38.73, \mathrm{p}=0.14)$ or to those that were HPV negative (OR $=3.52$, 95\% CI: 0.80 $15.52, \mathrm{p}=0.12)$. The decreased hrCL rate in women with combined hrHPV and non-hrHPV infections was nevertheless not significant as compared to those with single or multiple hrHPV infections ( $\mathrm{p}=0.06$ and $\mathrm{p}=0.34$, respectively).

When only hrHPV infections were considered, the women infected with 1 or 2 hrHPV genotypes had a significantly higher risk of developing hrCL with respect to the HPV-negative group ( $\mathrm{p}=0.004$ and $\mathrm{p}=0.02$, respectively; table 3; fig. 3). However, the risk for hrCL in women infected with $3+$ hrHPV genotypes decreased and showed no significant difference from those infected with multiple non-hrHPVs $(\mathrm{p}=0.68)$ or those who tested negative for HPV $(p=0.33)$. The OR of hrCL among women 


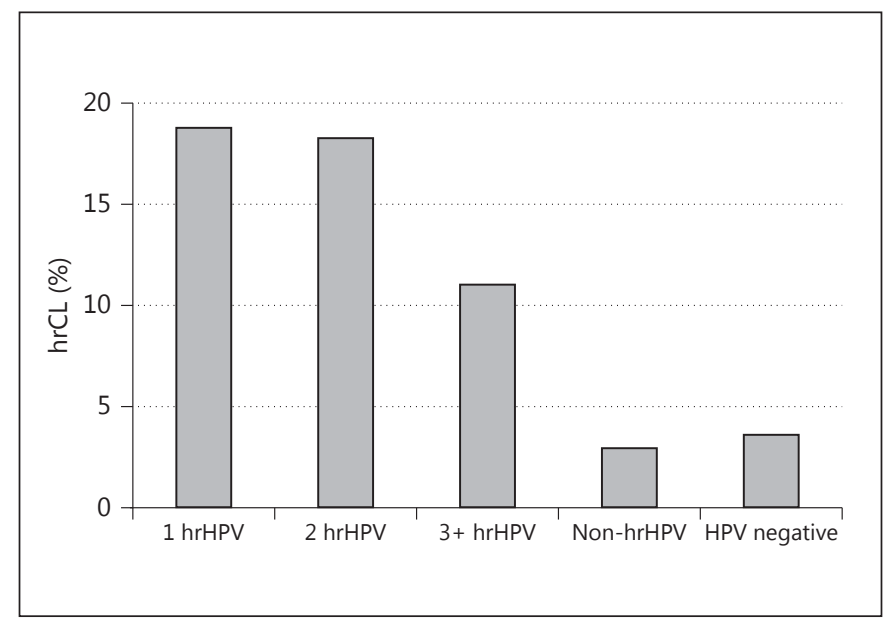

Fig. 3. Association of hrCL with number of hrHPV genotypes.

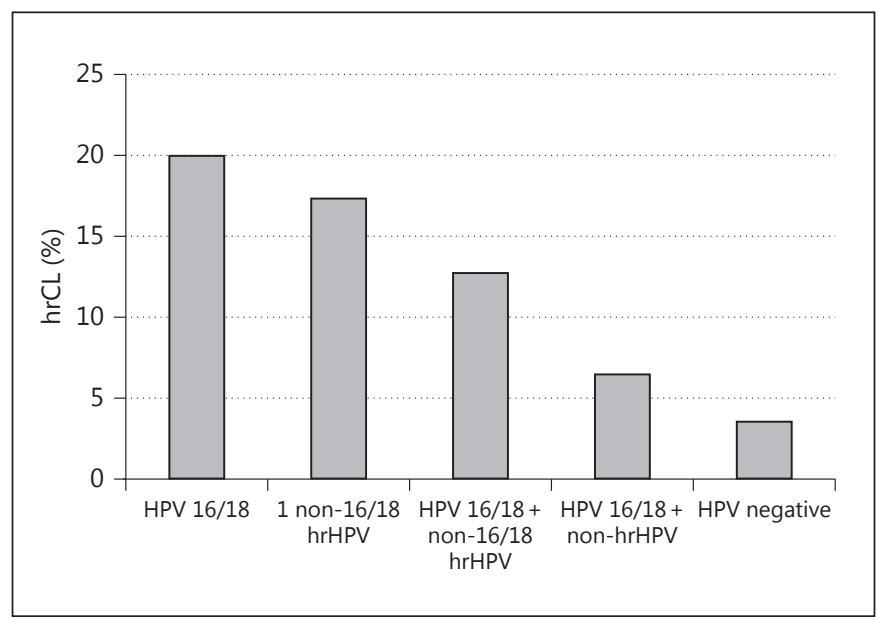

Fig. 4. Association of hrCL with HPV infection patterns related to HPV 16/18.
Fig. 5. ORs for HPV infection patterns associated with hrCL.

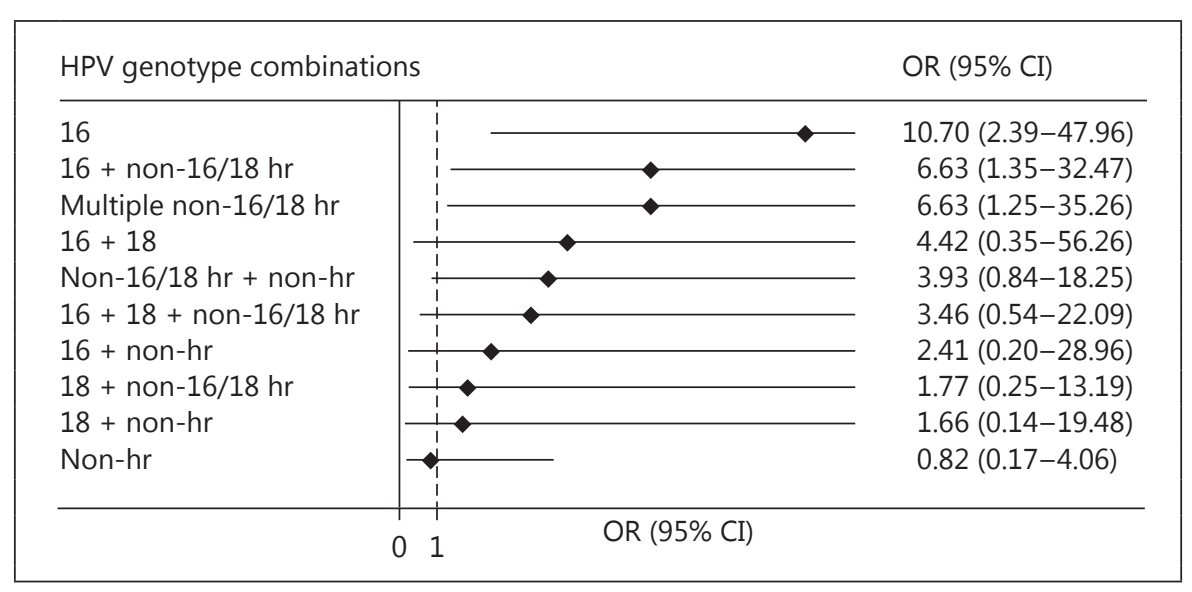

infected with 1, 2 or $3+$ hrHPV genotypes was statistically insignificant ( $\mathrm{p}=1.0$ for 1 vs. $2 \mathrm{hrHPV}, \mathrm{p}=0.43$ for 1 vs. $3+$ hrHPV and $p=0.53$ for 2 vs. 3 hrHPV). Despite being statistically insignificant, however, the risk for hrCL decreased with increasing number of co-infecting genotypes, especially when the infections involved $3+\mathrm{hrHPV}$ genotypes (fig. 3).

In light of the well-recognized role of HPV 16 and 18 in cervical dysplasia and cancer, we further analyzed the risk for hrCL in various infection patterns related to HPV 16 and 18 (table 4; fig. 4). Our analysis showed that hrCL was significantly associated with infection of HPV $16 / 18(20 \%, \mathrm{p}=0.005)$ and non-16/18 hrHPV genotypes $(17 \%, \mathrm{p}=0.01)$ as compared to the HPV-negative group (4\%). The rates of hrCL decreased in women who had HPV 16/18 infection combined with either non-16/18
hrHPV (13\%) or non-hrHPV (6\%) genotypes, with no significant difference with respect to that of the HPVnegative group ( $p=0.09$ and $p=0.62$, respectively). However, despite the downward trend observed in figure 4, the reduced hrCL rates in women with HPV 16/18 combined with non-16/18 hrHPV or non-hrHPV were not statistically significant compared to other infection patterns (table 4). The ORs (95\% CI) of various HPV infection patterns associated with hrCL are shown in figure 5 .

The rates of hrCL among women infected with 1,2 or $3+$ HPV species are summarized in table 5 . Women infected with 3+ HPV species appeared to have a reduced rate of hrCL (7\%) compared to infections with 1 (11\%) or 2 species (15\%). However, the differences were not statistically significant $(\mathrm{p}=0.26$ and $\mathrm{p}=0.08$, respectively). 
Table 5. Number of HPV species in cytology groups

\begin{tabular}{lclcc}
\hline Cytology group & HPV negative & 1 species & 2 species & $3+$ species \\
\hline hrCL & $2(4 \%)$ & $54(11 \%)$ & $28(15 \%)$ & $6(7 \%)$ \\
Non-hrCL & $53(96 \%)$ & $422(89 \%)$ & $153(85 \%)$ & $80(93 \%)$ \\
OR $(95 \%$ CI $)$ & & $3.39(0.80-14.31)$ & $4.85(1.12-21.05)$ & $1.99(0.39-10.22)$ \\
p value & & 0.1 & 0.02 & 0.48
\end{tabular}

hrCL = ASC-H, LSIL-H and HSIL. ORs (95\% CIs) were calculated with HPV negative as the reference group.

\section{Discussion}

In previous studies, multiple HPV infections were identified in $20-30 \%$ of the study cohorts [3-12, 20, 26$30]$. In the present study of underserved Hispanic women, infection with $2+$ genotypes was observed in $38.2 \%$ of the group. Women infected with hrHPV exhibited higher rates of hrCL than those infected with non-hrHPV regardless of single or multiple infections (fig. 4).

It is not clear in women with multiple HPV infections whether a competitive or cooperative interaction exists among the co-infecting genotypes. Several prior studies suggest a synergistic effect of multiple infections by HPV genotypes on oncogenesis [13-19], a conclusion that contradicts the findings of other investigators $[9,16,20-24]$. In the current study, we observed that single hrHPV infection carried the highest risk for hrCL. However, when women were co-infected with additional HPV genotypes, regardless of hrHPV or non-hrHPV, the hrCL rates were not higher as compared to the rates of the corresponding genotypes in single genotype infection or the projected hrCL rate of the combined genotypes. The results were consistent among various combinations of HPV infection patterns including hrHPV with another hrHPV, hrHPV with non-hrHPV, HPV 16/18 with non-16/18 hrHPV, or HPV 16/18 with non-hrHPV genotypes. Therefore, no additive or synergistic effect was identified in any of the various combinations of HPV infection patterns in our cohort. Conversely, we observed that multiple HPV infections were actually associated with lower rates of hrCL compared to the corresponding single HPV infection. For example, the hrCL rate decreased from 18.9 to $18.5 \%$ in women infected with 1 or $2 \mathrm{hrHPV}$ genotypes, respectively, and to $11 \%$ in those with $3+$ hrHPV genotypes (fig. 3 ). Reduced rates of hrCL were also observed in women with mixed infection of hrHPV with non-hrHPV (11.7\%; table 2), HPV 16/18 with non-16/18 hrHPV (12.7\%) or HPV $16 / 18$ with non-hrHPV genotypes (6.5\%; fig. 4$)$. In addi- tion, infection with $3+$ HPV species showed a reduced rate of hrCL (6.9\%) compared to infections with 1 or 2 species (11.5 and $15.4 \%$, respectively; table 5 ). The odds of having hrCL decreased in the women co-infected with additional genotypes as compared to those infected with a single genotype of HPV $16(\mathrm{OR}=10.70)$; the decrease was $38 \%$ with non-16/18 hrHPV (OR = 6.63), 59\% with HPV 18 $(\mathrm{OR}=4.42), 68 \%$ with HPV 18 and non-16/18 hrHPV $(\mathrm{OR}=3.46)$ and $77 \%$ with non-hrHPV $(\mathrm{OR}=2.41$; fig. 5$)$. Although the differences were not statistically significant in this relatively small cohort, the observed trend suggests the possibility of competitive interactions among certain HPV genotypes in individuals with multiple HPV infections. The aforementioned findings are in agreement with a recent study showing cross protection from progression to cervical cancer if co-infection with HPV types from the same species has occurred [25]. Our results are also in accordance with the established finding that women who are seropositive for both HPV 16 and HPV 6 are less likely to develop cervical cancer as compared to those who are seropositive only for HPV 16, a mechanism known as viral antagonism [31, 32]. Antagonistic interactions were also detected for combinations of HPV 16 and HPV 18 as well as HPV 16 and HPV 33 [31]. However, the mechanisms of the possible intergenotypic competition are likely more complex and may involve multiple key stages of the HPV infection process, including binding sites/receptors, utilization of host cell organelles, synthesis of viral DNA, insertion of viral DNA into the host genome and expression of viral proteins. A better understanding of the interactions among HPV genotypes is of great interest, not only for risk assessment of hrCLs in women with multiple HPV infections, but also for the development of the next generation of HPV vaccines by predicting the possible emerging genotypes that compete with those targeted by current vaccines (i.e. HPV 16 and 18). Further studies including larger cohorts are needed to elucidate the underlying mechanisms. 
In addition to the possible competitive effect among HPV genotypes, the host immune response should also be considered when evaluating the reduced risk for hrCL in women with multiple HPV infections. It has been shown that cervical HPV infection is higher in populations with impaired immune responses, such as HIV infection [33, 34] and cigarette smoking [35-37]. Natural HPV infections usually produce ineffective neutralizing antibodies in less than half of the infected women. In women with multiple HPV infections, the concurrent infection of multiple genotypes may collectively induce a more effective local or humoral immune response than that triggered by a single HPV infection. Alternatively, the weak and ineffective immunity generated by each HPV genotype may collectively provide an overall stronger immunity against HPV infection through antibody cross-reactions [36]. In support of this, cross-protective vaccine efficacy against infections and lesions associated with HPV 31, 33, and 45 was reported for both the bivalent and quadrivalent HPV vaccines [38]. The cross immunoreactions may effectively reduce or prevent viral invasion and replication, thus decreasing the risk for developing hrCLs.

Despite the observed trends in our present investigation, our results need to be interpreted cautiously as the study cohort is a high-risk population with a high HPV infection rate and it exhibits a different genotypic pattern from the general screening population in the United States [39]. In this respect, the findings from this study may not be applicable to other populations. In addition, this was a cross-sectional study without follow-up data evaluating the long-term effects of multiple HPV infections. Lastly, in this study we aimed to investigate the association between hrCL and various HPV infection patterns, without taking into account other factors such as
HIV status, tobacco use or sexual history, which may also be risk factors for hrCLs.

In conclusion, hrHPV infection was strongly associated with hrCL compared to infection with non-hrHPV regardless of single or multiple infections. Multiple HPV infections occurred frequently within the cohort, but they did not appear to carry an increased risk for hrCL compared to the women infected with the corresponding single HPV genotype. An additive or synergistic effect for hrCL was not identified in women infected with multiple HPV genotypes. A possibly reduced risk for hrCL was observed in women infected with various combinations of multiple HPV compared to their single-genotype-infected counterparts, although the differences were not statistically significant. The reasons underlying these findings are not yet known, but possible intergenotype competition or the mounting of a more effective immune response could be contributing factors. Additional studies in larger, more diverse populations are warranted to elucidate the role of multiple HPV infections in cervical dysplasia and cancer.

\section{Acknowledgments}

The authors thank Woo-Chul Moon, MD, PhD, for sponsoring the HPV genotyping tests, and Helen Chifotides, PhD, and Kathryn E. Stockbauer, $\mathrm{PhD}$, for editing of the paper. This study was not supported by any specific funding source.

\section{Disclosure Statement}

The authors of this study have no relevant financial interests to report.

\section{References}

-1 Pater MM, Dunne J, Hogan G, et al: Human papillomavirus types 16 and 18 sequences in early cervical neoplasia. Virology 1986;155: 13-18.

2 Biological Agents. Volume 100 B. A Review of Human Carcinogens. IARC Monogr Eval Carcinog Risks Hum. Lyon, IARC, 2012, vol 100B, pp 1-441.

$\checkmark 3$ Mendez F, Munoz N, Posso H, et al: Cervical coinfection with human papillomavirus (HPV) types and possible implications for the prevention of cervical cancer by HPV vaccines. J Infect Dis 2005;192:1158-1165.
-4 Vaccarella S, Franceschi S, Herrero R, et al: Clustering of multiple human papillomavirus infections in women from a population-based study in Guanacaste, Costa Rica. J Infect Dis 2011;204:385-390.

5 Thomas KK, Hughes JP, Kuypers JM, et al: Concurrent and sequential acquisition of different genital human papillomavirus types. J Infect Dis 2000;182:1097-1102.

6 Liaw KL, Hildesheim A, Burk RD, et al: A prospective study of human papillomavirus (HPV) type 16 DNA detection by polymerase chain reaction and its association with acquisition and persistence of other HPV types. J Infect Dis 2001;183:8-15.
7 Rousseau MC, Pereira JS, Prado JC, et al: Cervical coinfection with human papillomavirus (HPV) types as a predictor of acquisition and persistence of HPV infection. J Infect Dis 2001;184:1508-1517.

8 Chaturvedi AK, Myers L, Hammons AF, et al: Prevalence and clustering patterns of human papillomavirus genotypes in multiple infections. Cancer Epidemiol Biomarkers Prev 2005; 14:2439-2445.

$\checkmark 9$ Cuschieri KS, Cubie HA, Whitley MW, et al: Multiple high risk HPV infections are common in cervical neoplasia and young women in a cervical screening population. J Clin Pathol 2004;57:68-72. 
10 Dickson EL, Vogel RI, Bliss RL, et al: Multiple-type human papillomavirus (HPV) infections: a cross-sectional analysis of the prevalence of specific types in 309,000 women referred for HPV testing at the time of cervical cytology. Int J Gynecol Cancer 2013;23:12951302.

11 Huang LW, Chao SL, Chen PH, et al: Multiple HPV genotypes in cervical carcinomas: improved DNA detection and typing in archival tissues. J Clin Virol 2004;29:271-276.

12 Mejlhede N, Bonde J, Fomsgaard A: High frequency of multiple HPV types in cervical specimens from Danish women. APMIS 2009;117:108-114.

13 Bello BD, Spinillo A, Alberizzi P, et al: Cervical infections by multiple human papillomavirus (HPV) genotypes: prevalence and impact on the risk of precancerous epithelial lesions. J Med Virol 2009;81:703-712.

14 Fife KH, Cramer HM, Schroeder JM, et al: Detection of multiple human papillomavirus types in the lower genital tract correlates with cervical dysplasia. J Med Virol 2001;64:550 559.

15 Herrero R, Castle PE, Schiffman M, et al: Epidemiologic profile of type-specific human papillomavirus infection and cervical neoplasia in Guanacaste, Costa Rica. J Infect Dis 2005;191:1796-1807.

-16 Lee SA, Kang D, Seo SS, et al: Multiple HPV infection in cervical cancer screened by HPVDNAChip. Cancer Lett 2003;198:187192.

17 Pista A, Oliveira A, Verdasca N, et al: Single and multiple human papillomavirus infections in cervical abnormalities in Portuguese women. Clin Microbiol Infect 2011;17:941946.

18 Spinillo A, dal Bello B, Gardella B, et al: Multiple human papillomavirus infection and high grade cervical intraepithelial neoplasia among women with cytological diagnosis of atypical squamous cells of undetermined significance or low grade squamous intraepithelial lesions. Gynecol Oncol 2009;113:115119.
19 Trottier H, Mahmud S, Costa MC, et al: Human papillomavirus infections with multiple types and risk of cervical neoplasia. Cancer Epidemiol Biomarkers Prev 2006;15:12741280.

20 Chaturvedi AK, Katki HA, Hildesheim A, et al: Human papillomavirus infection with multiple types: pattern of coinfection and risk of cervical disease. J Infect Dis 2011;203:910920.

21 Levi JE, Kleter B, Quint WG, et al: High prevalence of human papillomavirus (HPV) infections and high frequency of multiple HPV genotypes in human immunodeficiency virus-infected women in Brazil. J Clin Microbiol 2002;40:3341-3345.

22 Rositch AF, Poole C, Hudgens MG, et al: Multiple human papillomavirus infections and type competition in men. J Infect Dis 2012; 205:72-81.

23 Wentzensen N, Nason M, Schiffman M, et al: No evidence for synergy between human papillomavirus genotypes for the risk of highgrade squamous intraepithelial lesions in a large population-based study. J Infect Dis 2014;209:855-864.

24 Wheeler CM, Hunt WC, Schiffman M, et al: Human papillomavirus genotypes and the cumulative 2-year risk of cervical precancer. J Infect Dis 2006;194:1291-1299.

25 Sobota RS, Ramogola-Masire D, Williams SM, et al: Co-infection with HPV types from the same species provides natural cross-protection from progression to cervical cancer. Infect Agent Cancer 2014;9:26.

26 Franco EL, Villa LL, Sobrinho JP, et al: Epidemiology of acquisition and clearance of cervical human papillomavirus infection in women from a high-risk area for cervical cancer. J Infect Dis 1999;180:1415-1423.

27 Plummer M, Schiffman M, Castle PE, et al: A 2 -year prospective study of human papillomavirus persistence among women with a cytological diagnosis of atypical squamous cells of undetermined significance or low-grade squamous intraepithelial lesion. J Infect Dis 2007;195:1582-1589.

28 Vaccarella S, Franceschi S, Snijders PJ, et al: Concurrent infection with multiple human papillomavirus types: pooled analysis of the IARC HPV Prevalence Surveys. Cancer Epidemiol Biomarkers Prev 2010;19:503-510.
29 Bauer HM, Ting Y, Greer CE, et al: Genital human papillomavirus infection in female university students as determined by a PCRbased method. JAMA 1991;265:472-477.

30 Herrero R, Hildesheim A, Bratti C, et al: Population-based study of human papillomavirus infection and cervical neoplasia in rural Costa Rica. J Natl Cancer Inst 2000;92:464-474.

31 Luostarinen T, af Geijersstam V, Bjorge T, et al: No excess risk of cervical carcinoma among women seropositive for both HPV16 and HPV6/11. Int J Cancer 1999;80:818-822.

32 Silins I, Wang Z, Avall-Lundqvist E, et al: Serological evidence for protection by human papillomavirus (HPV) type 6 infection against HPV type 16 cervical carcinogenesis. J Gen Virol 1999;80(pt 11):2931-2936.

33 Chaturvedi AK, Dumestre J, Gaffga AM, et al: Prevalence of human papillomavirus genotypes in women from three clinical settings. J Med Virol 2005;75:105-113.

34 Levi JE, Fernandes S, Tateno AF, et al: Presence of multiple human papillomavirus types in cervical samples from HIV-infected women. Gynecol Oncol 2004;92:225-231.

- 35 Arcavi L, Benowitz NL: Cigarette smoking and infection. Arch Intern Med 2004; 164 : 2206-2216.

36 Poppe WA, Ide PS, Drijkoningen MP, et al: Tobacco smoking impairs the local immunosurveillance in the uterine cervix. An immunohistochemical study. Gynecol Obstet Invest 1995;39:34-38.

37 Sedlacek TV: Advances in the diagnosis and treatment of human papillomavirus infections. Clin Obstet Gynecol 1999;42:206-220.

38 Malagon T, Drolet M, Boily MC, et al: Crossprotective efficacy of two human papillomavirus vaccines: a systematic review and metaanalysis. Lancet Infect Dis 2012;12:781-789.

39 Zhou H, Schwartz MR, Coffey DM, et al: Genotype-specific prevalence and distribution of human papillomavirus genotypes in underserved Latino women with abnormal $\mathrm{Pa}$ panicolaou tests. J Am Soc Cytopathol 2014; 4:42-48. 